\title{
Calidad Educativa Percibida por los Alumnos y Satisfacción Laboral Docente de la Universidad Alas Peruanas Filial Jaén
}

Educational Quality perceived by Students and Job Satisfaction at Alas Peruanas University Jaén

${ }^{1}$ Delicia Liliana Bazán Tantaleán

\section{RESUMEN}

Este trabajo se realizó en la Universidad Alas Peruanas Filial Jaén, con el objetivo de determinar la relación que existe entre la calidad educativa percibida por los alumnos y la satisfacción laboral del docente en el año académico 2014.

El tipo de investigación fue correlacional, donde se determinó la relación que existe entre las variables Calidad Educativa y Satisfacción Laboral docente y sus dimensiones. Además de un análisis situacional de la calidad de servicio y satisfacción laboral. El diseño de investigación es no experimental y transversal.

E1 96.9\% de los estudiantes percibe la Calidad Educativa como medianamente eficiente y solo 3.1\% la considera deficiente, con énfasis en la dimensión de gestión universitaria. Así mismo el 89.1\% de los docentes se encuentran regularmente satisfechos en su labor docente y solo el $10.1 \%$ el grado de satisfacción es bajo, la dimensión con menor calificativo es Labor Docente. Se concluyó que se acepta la hipótesis de relación de las variables Calidad Educativa y Satisfacción Laboral Docente, empleando el Test de Chi-cuadrado de Pearson.

Palabras clave: Formación profesional, Gestión universitaria.

\begin{abstract}
This work was carried out at the Alas Peruanas University, Jaén Branch, with the objective of the research was to determine the relationship between the educational quality perceived by the students and the teacher's job satisfaction in the academic year 2014.

The type of research was correlational, to determine the relationship between the variables Educational Quality and Teacher Satisfaction and their dimensions. In addition to a situational analysis of the quality of service and job satisfaction. The research design was non-experimental and cross-sectional.

A $96,9 \%$ of the students perceive the Educational Quality as moderately efficient and only 3,1\% consider it deficient, with emphasis on the University Management dimension. Likewise, 89,1\% of teachers are regularly satisfied in their teaching work and only a 10,1\%, the degree of satisfaction is low, the dimension less qualifying is Labor Teaching. It was concluded that the hypothesis of relation of the variables Educational Quality and Teaching Satisfaction is accepted, using the Pearson Chi-square test.
\end{abstract}

Keywords: Vocational training, University management.

'Universidad Nacional de Jaén. Jaén, Cajamarca, Perú 


\section{INTRODUCCIÓN}

La calidad es el conjunto de características inherentes a un producto o servicio que cumple los requisitos para satisfacer las necesidades preestablecidas. Así una carrera profesional de calidad define claramente su misión o propósito en función de sus grupos de interés, estos propósitos abarcan las actividades confiadas por la sociedad(SINEACE, 2008).

La universidad peruana no es ajena a esta realidad y para insertarse en un escenario tan competitivo necesita contar con estrategias que la conduzcan a superar estos retos: establecer la igualdad de condiciones de acceso a los estudios, vincularse con los grupos de interés, desarrollar capital humano, es decir, atraer y retener al personal con talento comprometido en la enseñanza e investigación, lograr una formación de estudiantes ligados al desarrollo del pensamiento crítico e independiente de calidad (Viaña, Vargas, \& Golergant, 2007).

En la Universidad de Cartagena, se realizó un estudio sobre Análisis de la calidad en el servicio y satisfacción de los estudiantes de Ciencias Económicas mediante un modelo de ecuaciones estructurales, con el objetivo de evaluar la calidad del servicio ofrecido por la universidad, en las distintas unidades académicas de la Facultad de Ciencias Económicas, mediante las percepciones y las expectativas de los estudiantes, haciendo uso de ecuaciones estructurales (Vergara \& Quesada, 2011). La población objeto de análisis la conformaron estudiantes de cada uno de sus programas: Economía, Administración Industrial, Administración de Empresas y Contaduría Pública y la muestra que equivale a 1,877 personas. En el estudio, se utilizó una adaptación del modelo propuesto por Oh (1999) y la escala de medición propuesta en el modelo SERVQUAL para analizar la calidad en el servicio. Concluyeron que entre las correlaciones obtenidas se pudieron destacar las percepciones con un alto índice de correlación con respecto a dos variables, en las cuales influyen significativamente, además para poder aumentar la calidad del servicio académico percibido se debe hacer énfasis en las percepciones de los servicios que se brinda a los estudiantes. También aumentar la calidad del servicio académico tendrá un doble efecto positivo sobre la satisfacción de los estudiantes de la facultad, incrementando el valor percibido por el estudiante y su satisfacción. Luego, por la relación que existe entre el valor percibido por el estudiante y la satisfacción del mismo, se observa que si aumenta el valor percibido por el estudiante, aumentará su satisfacción hacia los servicios que ofrece la institución. En un estudio de referencia realizado por Oh (1999) se llega a una conclusión similar.

(Sosa, 2011), realizó un trabajo de investigación sobre la Calidad en los servicios Educativos en la Universidad de San Martín de Porres Filial Norte. Esta investigación al mismo tiempo colaboró con los objetivos de la casa superior de estudios, en la planificación de sus estrategias, actividades y acciones. Se midió la calidad utilizando el cuestionario SERVQUAL (Service Quality), adaptado al sector educativo, con alto nivel de fiabilidad y validez con 0.94 de coeficiente alfa Combrach. Las encuestas se realizaron a una muestra de 373 alumnos y al personal administrativo que está relacionado con todos los servicios de la institución y que interactúa con los alumnos. Las conclusiones fueron que las expectativas de los estudiantes para cada uno de los indicadores fueron muy altas. La dimensión de empatía tuvo la expectativa más alta y la dimensión de tangibles obtuvo la expectativa más baja. Los indicadores, amplitud de aula, limpieza de ambientes y docentes con dominio de la asignatura tuvieron las más alta percepciones. Las instituciones de educación superior deben medir la calidad de sus servicios periódicamente, con la finalidad de conocer sus fortalezas y debilidades para que posteriormente elaboren estrategias de mejora. 
El presente trabajo de investigación tuvo como objetivo conocer la percepción de los estudiantes frente a la calidad educativa de la UAP, al no existir antecedentes de otras investigaciones en la misma universidad, que permita conocer más de cerca, cuáles podrían ser esas necesidades que requieren atención, a fin de evaluar y realizar las acciones pertinentes encaminadas a lograr la mejora de la calidad, es decir con el principal interés de conocer los aspectos positivos y negativos a través de la construcción de un instrumento de medición. Además nos permitió conocer los niveles de satisfacción laboral y determinar aquellas áreas de mayor y menor satisfacción, de tal manera que genere indicadores de mejora, así mismo su relación con la calidad educativa, que permita contribuir a que la institución reciba una retroalimentación que le ayude a optimizar el servicio educativo y la satisfacción laboral.

\section{MATERIALES Y MÉTODOS}

El tipo de investigación fue correlacional, donde se determinó la relación que existe entre la variable Calidad de Servicio y Satisfacción Laboral y sus dimensiones. El diseño de investigación fue No experimental transversal, mide la relación de las variables en un momento único en el tiempo. El Método de investigación fue de Comparación y las técnicas utilizadas fueron encuestas y análisis de contenido.

La población objeto de análisis estuvo integrada por todos los estudiantes (922) y docentes (55) de la Universidad Alas Peruanas Filial Jaén de los programas: Ciencias Contables y Financieras, Ingeniería Civil, Administración y Negocios Internacionales, Derecho, Administración y Obstetricia. La muestra de docentes es la misma que la población (55), y la muestra objetiva de alumnos es una muestra probabilística teniendo como resultado 350 estudiantes, cuyas unidades de análisis o elementos muéstrales se eligió aleatoriamente.

Larecolección de datos se realizó mediante la aplicación de instrumentos denominado "Escala de calidad educativa percibida por los alumnos de la Universidad Alas Peruanas Filial Jaén" y el instrumento "Escala de satisfacción laboral percibida por los docentes de la Universidad Alas Peruanas Filial Jaén".

Los instrumentos fueron elaborados por el autor y validado por el instrumento de opinión de expertos; el primer instrumento denominado "Escala de calidad educativa percibida por los alumnos de la Universidad Alas Peruanas Filial Jaén", fue diseñada en 47 ítems y dividido en 4 dimensiones: Gestión Universitaria que contiene 8 ítems, Formación Profesional por 17 ítems, Investigación y Proyección Social por 7 ítems, Servicios de Apoyo para la formación profesional por 15 ítems, se aplicó el método de recolección de datos empleando un cuestionario de escala tipo Likert, con los criterios de valoración: Muy deficiente, Deficiente, Medianamente eficiente, Eficiente, Sobresaliente los mismos que tienen relación con los indicadores de la variable: Calidad educativa. Como se muestra en la tabla 1 .

Tabla 1: Escala general de la variable Calidad educativa.

\begin{tabular}{|c|c|c|c|}
\hline $\begin{array}{c}\text { Valoración de } \\
\text { Respuesta }\end{array}$ & $\begin{array}{c}\text { Valoración de Variable : Calidad } \\
\text { Educativa }\end{array}$ & Puntaje & Rango \\
\hline Nunca & Muy deficiente & 0 & $\begin{array}{c}{[00-37} \\
\text { puntos }]\end{array}$ \\
\hline Pocas veces & Deficiente & 1 & $\begin{array}{c}{[38-74} \\
\text { puntos }]\end{array}$ \\
\hline Algunas veces & Medianamente eficiente & 2 & $\begin{array}{c}{[75-111} \\
\text { puntos }]\end{array}$ \\
\hline Frecuentemente & Eficiente & 3 & $\begin{array}{c}{[112-148} \\
\text { puntos }]\end{array}$ \\
\hline Siempre & Sobresaliente & 4 & $\begin{array}{c}{[149-184} \\
\text { puntos }]\end{array}$ \\
\hline
\end{tabular}

Fuente: Elaboración propia.

Para determinar la Satisfacción Laboral, se aplicó el instrumento "Escala de Satisfacción Laboral percibida por los docentes de la Universidad Alas Peruanas Filial Jaén", diseñada en 40 ítems y dividida en 4 dimensiones: Labor Docente - Gestión que contiene 9 ítems, Labor de enseñanza y reconocimiento con 10 ítems, 
Relaciones profesionales en la Labor de investigación y proyección social con 9 ítems y Condiciones Laborales con 12 ítems; se aplicó el método de recolección de datos empleando un cuestionario de escala tipo Likert, con los mismos criterios de valoración, como se muestra en la tabla 2.

Tabla 2: Escala General de la Variable Satisfacción Laboral.

\begin{tabular}{|c|c|c|c|}
\hline $\begin{array}{c}\text { Valoración de } \\
\text { Respuesta }\end{array}$ & $\begin{array}{c}\text { Valoración de } \\
\text { Variable : } \\
\text { Satisfacción } \\
\text { laboral }\end{array}$ & Puntaje & Rango \\
\hline Nunca & Muy baja & 0 & $\begin{array}{c}{[00-23} \\
\text { puntos }]\end{array}$ \\
\hline Pocas veces & Baja & 1 & $\begin{array}{c}{[24-46} \\
\text { puntos }]\end{array}$ \\
\hline Algunas veces & Regular & 2 & $\begin{array}{c}{[47-69} \\
\text { puntos }]\end{array}$ \\
\hline Frecuentemente & Alta & 3 & $\begin{array}{c}{[70-92} \\
\text { puntos }]\end{array}$ \\
\hline Siempre & Muy alta & 4 & $\begin{array}{c}{[93-116} \\
\text { puntos }]\end{array}$ \\
\hline
\end{tabular}

Fuente: Elaboración propia.

\section{RESULTADOS}

\section{Calidad Educativa}

La tabla 3, muestra el análisis de los puntajes obtenidos de la percepción de los alumnos en el estudio de la Calidad Educativa y reveló que el promedio de 90,37 de los estudiantes percibe la calidad educativa con una calificación medianamente eficiente, teniendo en cuenta las cuatro dimensiones trabajadas de Gestión Universitaria, Formación profesional, Investigación - proyección social y Servicios de apoyo para la formación profesional.
Tabla 3: Estadísticas Descriptivas de Calidad Educativa en Opinión de los Estudiantes de la Universidad Alas Peruanas Filial Jaén.

\begin{tabular}{|c|c|}
\hline 9 läŕr kët'd & $/ ![\mathbf{1 5} ! 5$ \\
\hline$\overline{\mathrm{b}}$ ë A $\mathrm{Al}^{\prime} \mathbf{a} \mathrm{a}$ & يجيق \\
\hline $\mathrm{t}$ śń $^{\prime} \Psi \mathbf{a}$ & t \\
\hline a śrí & يقى \\
\hline 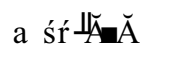 & لا لأهt \\
\hline 5 ślō & كهىوبو \\
\hline 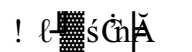 & .074 \\
\hline / j’iralt & .383 \\
\hline a 轞口 & ي يـ \\
\hline a As & ي لاًا \\
\hline
\end{tabular}

Fuente: Instrumento escala valorativa para medir la Calidad Educativa en opinión de los estudiantes de la Universidad Alas Peruanas Filial Jaén.

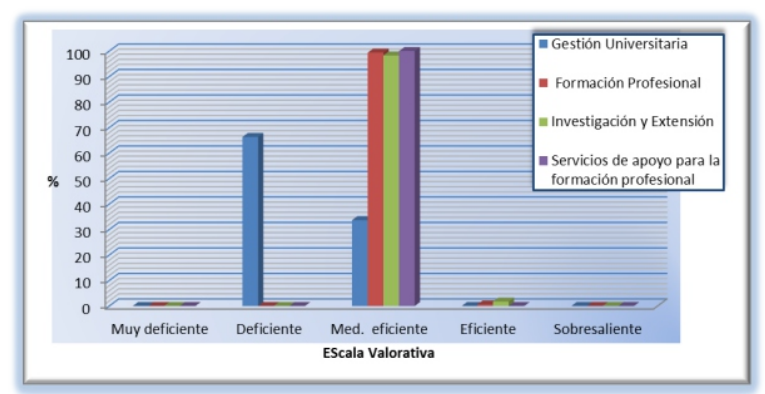

Figura 1: Niveles de Calidad Educativa por dimensiones en opinión de los estudiantes de la Universidad Alas Peruanas Filial Jaén -2014.

Fuente: Instrumento escala valorativa para medir la Calidad Educativa en opinión de los estudiantes de la Universidad Alas Peruanas Filial Jaén-Perú.

La figura 1 muestra que el $66.3 \%$ de los estudiantes de la UAP percibe la Calidad Educativa en la dimensión de Gestión Universitaria como deficiente y el $33.7 \%$ considera medianamente eficiente, es decir tienen poco conocimiento del plan estratégico y operativo de su escuela profesional; así mismo considera la coordinación entre las áreas académicas y administrativas como deficiente; el 
sistema de gestión de calidad y los programas de motivación e incentivo para estudiantes no está funcionando correctamente. Esto se debe, a que la participación de los estudiantes en la elaboración de los instrumentos de gestión es baja, por tal motivo su desconocimiento, falta concientización en el sistema de implementación del sistema de gestión de calidad y a que no se sienten involucrados. En cuanto a los programas de incentivos, los estudiantes con calificativos altos son beneficiados con becas de estudio, pero son pocos los becarios.

Además también se puede apreciar en la figura 1, que el 94, 4.3\% de los estudiantes percibe la Calidad Educativa, en la dimensión de Formación profesional, medianamente eficiente y el $0.6 \%$ eficiente. La formación profesional que involucra las subdimensiones de proyecto educativo - currículo, las estrategias de enseñanza aprendizaje y desempeño docente son considerados en término medio por los estudiantes. Los criterios de frecuencia en la elaboración de tesis, estrategias de enseñanza, sistema de evaluación, cumplimiento del silabo, tutoría y especialidad del docente, entre otros, son considerados medianamente eficientes. Esta calificación se debe probablemente porque la horas practicas no se cumplen, por la falta de laboratorio o por falta de implementación de los laboratorios, se tiene que tercerisar las prácticas en otras universidades, así mismo en las estrategias usadas por el docente para desarrollar la capacidad de investigación, es uno de los aspectos que han considerado deficiente, por lo que puede y debe ser mejorado.

La figura 1, también muestra que el 98.3\% de los estudiantes percibe la Calidad Educativa, en la dimensión de Investigación y proyección social, Medianamente eficiente y el $1.7 \%$ Eficiente. Esto involucra el sistema de evaluación de los proyectos de investigación, la frecuencia de participación de los estudiantes, los eventos de difusión y publicación de los trabajos de investigación, proyección social y extensión universitaria, incluyendo los procedimientos de adquisición de los derechos de propiedad intelectual sobre lo creado como resultado de la investigación, la participación de los estudiantes en el año 2014 se han presentado 3 trabajos de investigación, por lo que se debe mejorar este aspecto. No existe información de los docentes.

En la figura 1 también se observa en, que el 100\% de los estudiantes de la UAP percibe la Calidad Educativa, en la dimensión de Servicios de apoyo para la formación profesional, medianamente eficiente. Los servicios de bienestar universitario también son considerados medianamente eficientes. Es decir la comodidad, seguridad, limpieza y el equipamiento de la infraestructura para el desarrollo de la enseñanza-aprendizaje, investigación, extensión universitaria y proyección social y el programa implementación para su mantenimiento, renovación y ampliación son medianamente eficiente según los estudiantes encuestados. Así mismo la actualización e implementación de la biblioteca y equipos tecnológicos, como los programas de bienestar universitario son considerados por los estudiantes medianamente eficientes. El campus universitario es propio de la institución, cuenta con áreas verdes y centro de recreación, actualmente tiene 5 pisos. Así mismo los servicios de bienestar universitario como el servicio de atención médica y psicológica se encuentran poco implementados, pero las actividades deportivas y culturales se mantienen dinámicas.

\section{Satisfacción Laboral Docente}

La tabla 4 muestra un análisis de los puntajes obtenidos del instrumento aplicado a los docentes para el estudio de Satisfacción laboral docente, reveló que obtuvieron un promedio de 56.82 puntos con una variación de 1.827, significa que los docentes se consideran regularmente satisfechos con su labor docente, 
teniendo en cuenta las cuatro dimensiones en estudio que son: Labor Docente-Gestión, Labor de enseñanza y reconocimiento, relaciones profesionales en la labor de investigación y proyección social y condiciones laborales.

TABLA 4: Estadísticas descriptivas de los Puntajes Obtenidos de Satisfacción Docente en opinión a los docentes de la Universidad Alas Peruanas Filial Jaén -2014.

\begin{tabular}{lc} 
Estadísticos & SATISFACCION DOCENTE \\
\hline $\mathrm{N}$ Válidos & 55 \\
$\quad$ Perdidos & 0 \\
Media & 56.82 \\
Mediana & 57.00 \\
Desv. típ. & 1.827 \\
Asimetría & -.156 \\
Curtosis & -.672 \\
Mínimo & 53 \\
Máximo & 61 \\
\hline
\end{tabular}

Fuente: Instrumento escala valorativa para medir la Satisfacción Docente en opinión de los docentes de la Universidad Alas Peruanas - Filial Jaén-Perú.

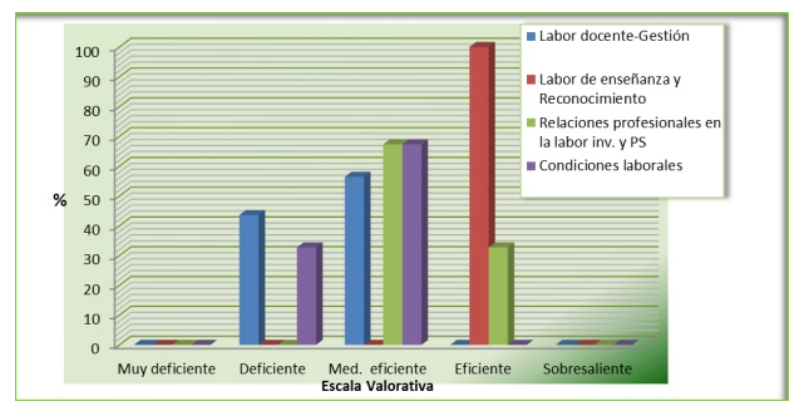

Figura2: Niveles de Satisfacción Docente en Opinión de Docentes de la Universidad Alas Peruanas Filial Jaén-Perù-2014.

En la figura 2, muestra que el 56,4\% de los docentes se encuentra regularmente satisfecho en la dimensión de Labor docente-Gestión y el 43,6\% se encuentra en un nivel de satisfacción bajo. Esto refleja la misma problemática que manifestaron los estudiantes, al no tener participación en la elaboración de los instrumentos de gestión, al no sentirse involucrados con el sistema de gestión de calidad y percibir una baja coordinación entre las áreas académicas y administrativas en la ejecución de los procesos; los resultados también indican que algunos docentes desconocían los objetivos, políticas y lineamientos estratégicos por tal motivo el grado de satisfacción que sienten los docentes es regular y bajo.

La figura 2, también se observa que el 100\% de los docentes, siente un alto grado de satisfacción por su Labor de enseñanza y reconocimiento, es decir en su labor de impartir clases, en el proceso de enseñanza - aprendizaje que aplican, en la distribución de horas lectivas, de investigación, de proyección y extensión universitaria. Además el reconocimiento a su labor de enseñanza y la evaluación al desempeño docente son altos. En la UAP cada fin de ciclo se reconoce al docente con una constancia de felicitación, por obtener los más altos resultados en la evaluación docente, realizada por la escuela profesional, además se ofrece el $25 \%$ y $50 \%$ de descuento en estudios de posgrado a los docentes según evaluación.

La figura 2, muestra además que el $67.3 \%$ de los docentes, se siente regularmente satisfecho por las Relaciones profesionales para la Labor de investigación y proyección social y el $32.7 \%$ altamente satisfecho, es decir el trabajo en equipo, la reunion de coordinación para elaborar proyectos de investigación y proyección social es considerado regular en grado de satisfacción. Esto se debe a que los docentes en su mayoría a tiempo parcial no facilita las relaciones profesionales para la coordinación y elaboración de proyectos interdisciplinarios, además la oficina de investigación no tiene plena autonomía, ya que depende de la sede central y el apoyo que brinda la UAP no es dinámico.

La figura 2, también muestra que el $67.3 \%$ de los docentes, se siente regularmente satisfechos por las Condiciones Laborales que ofrece la UAP y el $32.7 \%$ con un grado de satisfacción bajo, es 
decir las condiciones de autonomía como docente en el desarrollo de la asignatura, la remuneraciones, la estabilidad laboral, los ambientes de trabajo, el uso de la tecnología para el desarrollo de clases, la limpieza y orden de los ambientes, la implementación y actualización de la biblioteca y los programas de bienestar universitario es percibido por los docentes de manera regular y baja. El ítem con menor puntaje fue de satisfacción por las remuneraciones y la estabilidad laboral, esto se debe a que las remuneraciones de los docentes son categorizadas según el grado de formación, los contratos son temporales para la mayoría de los docentes, los ambientes de trabajo no todos cuentan con medios tecnológicos audiovisuales, y los programas de bienestar universitario no son muy difundidos.

\section{Estadística Inferenciales: Calidad Educativa y Satisfacción Laboral Docente}

La tabla 5, muestra el valor de Chi-cuadrado de Pearson sobre la independencia de las dos variables; el valor (muestral) del estadístico es 6.254; los grados de libertad (gl) son 2 y la sig. Asintótica bilateral es de 0.072 (mayor que $\geq$ 0.05), significa que aceptamos la hipótesis de relación de dependencia de las variables de Calidad Educativa con énfasis en el nivel (Medianamente eficiente) y Satisfacción Laboral Docente en el nivel (Regular).

Asimismo, contrastada frente a los resultados del modelo teórico Chi-cuadrado, el valor límite crítico es de 5.991 entonces valor Chi-cuadrado obtenido es mayor al valor crítico $(5.991<6.254)$, indicando que los resultados observados tienen un $95 \%$ de probabilidad de coincidencia respecto de los resultados teóricos esperados, es decir se acepta la hipótesis alternante.
Tabla 5: Niveles de calidad educativa y satisfacción laboral docente. Pruebas de chicuadrado.

\begin{tabular}{|l|r|r|r|}
\hline & \multicolumn{1}{|c|}{ Valor } & gl & Sig. asintótica (bilateral) \\
\hline Chi-cuadrado de Pearson & $6.254(\mathrm{a})$ & 2 & 0.072 \\
Razón de verosimilitudes & 5.020 & 2 & 0.081 \\
N de casos válidos & 350 & & \\
\hline
\end{tabular}

a 2 casillas (33.3\%) tienen una frecuencia esperada inferior a 5 . La frecuencia mínima esperada es 0.19 .

La tabla 6, muestra el valor de Chi-cuadrado de Pearson sobre la independencia de las dos variables; el valor (muestral) del estadístico es

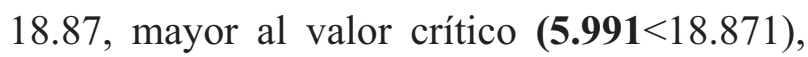
indicando que los resultados observados tienen un $95 \%$ de probabilidad de coincidencia respecto de los resultados teóricos esperados. Esta información de análisis de inferencia probabilística, verifica que existen indicios de una relación entre ambas dimensiones: Gestión Universitaria y Labor Docente-Gestión.

Así mismo la tabla 6, muestra los grados de libertad (gl) 2 y la sig. Asintótica bilateral de 0,080 (mayor que $\geq 0.05$ ), lo que permite aceptar la hipótesis de relación de dependencia de las dimensiones Gestión Universitaria en su nivel (deficiente) y la dimensión: Labor docenteGestión en su nivel (Regular).

Tabla 6: Niveles de calidad educativa: dimensión gestión universitaria y satisfacción laboral: dimensión labor docente-gestión. Pruebas de chi-cuadrado.

\begin{tabular}{|c|c|c|c|}
\hline & ë $\AA_{\text {-in }}$ & & 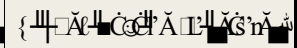 \\
\hline / Ш cuadrado de Pearson $_{\text {chad }}$ & | & 9 & 8.080 \\
\hline 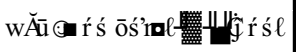 & لاله & 9 & 8.078 \\
\hline 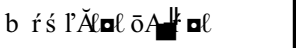 & عيول & & \\
\hline
\end{tabular}

a 2 casillas $(33.3 \%)$ tienen una frecuencia esperada inferior a 5. La frecuencia mínima esperada es 0.41 .

La tabla 7, muestra el valor de Chi-cuadrado de Pearson sobre la independencia de las dos variables; el valor (muestral) del estadístico es 


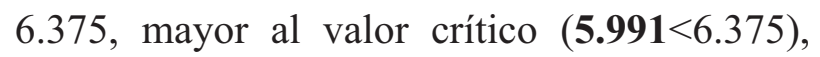
indicando que los resultados observados tienen un $95 \%$ de probabilidad de coincidencia respecto de los resultados teóricos esperados. Esta información de análisis de inferencia probabilística, verifica que existen indicios de una relación entre ambas dimensiones: Formación Profesional y Labor de enseñanza y reconocimiento.

Así mismo la tabla 7, muestran los grados de libertad (gl) 2 y la sig. Asintótica bilateral de 0.829 (mayor que $\geq 0.05$ ), lo que permite aceptar la hipótesis de relación de dependencia de las dimensiones Formación Profesional en su nivel (Medianamente Eficiente) y la dimensión Labor de enseñanza y reconocimiento en su nivel (Alta).

Tabla 7: Niveles de calidad educativa: dimensión formación profesional y satisfacción laboral: dimensión: labor de enseñanza y reconocimiento. Pruebas de chi-cuadrado.

\begin{tabular}{|l|r|r|r|}
\hline & \multicolumn{1}{|c|}{ Valor } & gl & Sig. asintótica (bilateral) \\
\hline Chi-cuadrado de Pearson & $6.375(\mathrm{a})$ & 2 & 0.829 \\
Razón de verosimilitudes & .686 & 2 & 0.710 \\
N de casos válidos & 350 & & \\
\hline
\end{tabular}

a 3 casillas $(50.0 \%)$ tienen una frecuencia esperada inferior a 5. La frecuencia mínima esperada es 0.10 .

La tabla 8, muestra el valor de Chi-cuadrado de Pearson sobre independencia de las dos variables; el valor (muestral) del estadístico es

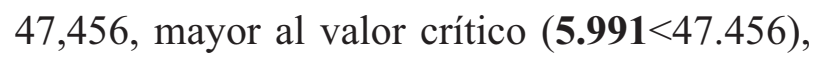
indicando que los resultados observados tienen un $95 \%$ de probabilidad de coincidencia respecto de los resultados teóricos esperados. Esta información de análisis de inferencia probabilística, verifica que existen indicios de una relación entre ambas dimensiones: Investigación, proyección social y Relaciones profesionales en la Labor de investigación y proyección social.
Así mismo la tabla 8, muestra los grados de libertad (g1) 2 y la sig. Asintótica bilateral de 0.620 (mayor que $\geq 0.05$ ), lo que permite aceptar la hipótesis de relación de dependencia de las dimensiones: Investigación y proyección social en su nivel (Medianamente eficiente) y la dimensión Relaciones profesionales en la Labor de investigación y proyección social en su nivel (Regular).

Tabla 8: Niveles de calidad educativa: dimensión investigación y proyección social y satisfacción laboral dimensión: relaciones profesionales en la labor de investigación y proyección social. Pruebas de chi-cuadrado.

\begin{tabular}{|c|c|c|c|}
\hline & $\ddot{~ e ̈ ~}$ & & 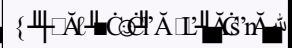 \\
\hline$/ \Psi_{\text {cuadrado de Pearson }}$ & 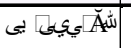 & 9 & 8.620 \\
\hline wầi & لآييَ بو & 9 & +.524 \\
\hline b ŕś l'Ălal ō & بse & & \\
\hline
\end{tabular}

a 2 casillas (33.3\%) tienen una frecuencia esperada inferior a 5. La frecuencia mínima esperada es 0.31 .

La tabla 9, muestra el valor de Chi-cuadrado de Pearson sobre la independencia de las dos variables; el valor (muestral) del estadístico es

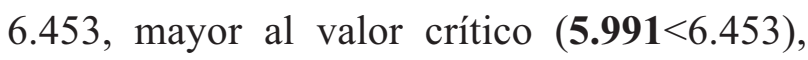
indicando que los resultados observados tienen un $95 \%$ de probabilidad de coincidencia respecto de los resultados teóricos esperados. Esta información de análisis de inferencia probabilística, verifica que existen indicios de una relación entre ambas dimensiones: servicios de apoyo en la Formación Profesional y Condiciones laborales.

Así mismo la tabla 9, muestra los grados de libertad (gl) 2 y la sig. Asintótica bilateral de 0.6590 (mayor que $\geq 0.05$ ), lo que permite aceptar la hipótesis de relación de dependencia de las dimensiones: servicios de apoyo en la Formación Profesional en su nivel (Medianamente eficiente) y la dimensión Condiciones laborales en su nivel (Regular). 
Tabla 9: Niveles de Calidad Educativa: Dimensión Servicios de apoyo para la formación profesional y Satisfacción laboral en la Dimensión: Condiciones laborales. Pruebas de chi-cuadrado.

\begin{tabular}{|l|r|r|r|}
\hline & \multicolumn{1}{|c|}{ Valor } & gl & Sig. asintótica (bilateral) \\
\hline Chi-cuadrado de Pearson & $6.453(\mathrm{a})$ & 2 & 0.659 \\
Razón de verosimilitudes & .576 & 2 & 0.561 \\
N de casos válidos & 350 & & \\
\hline
\end{tabular}

a 3 casillas $(50.0 \%)$ tienen una frecuencia esperada inferior a 5. La frecuencia mínima esperada es 0.10 .

\section{DISCUSIÓN}

Existe evidencia estadística que afirma con un 95\% de confianza, que la correlación es verdadera entre las dos variables, calidad educativa y satisfacción laboral docente, cumpliéndose la hipótesis general; es decir se puede afirmar que a medida que se mejora la satisfacción laboral docente, la calidad educativa mejora o viceversa. Son los docentes también protagonistas de la calidad, si están satisfechos con su labor, remuneración, estabilidad, reconocimiento, etc. ayudarán al cumplimiento de los estándares de calidad y se observará una calidad educativa eficiente.

En el análisis del diagnóstico situacional de la UAP, en relación a la primera variable, calidad educativa, es percibida por los estudiantes de medianamente eficiente en un $96.9 \% \mathrm{y}$ deficiente en un $3,1 \%$, es decir no cubre completamente las expectativas de los estudiantes, principalmente en la dimensión gestión universitaria que tuvo un calificativo de deficiente con un porcentaje de $66.3 \%$, el alumno no se siente involucrado con el sistema de gestión de calidad, existe poca satisfacción con la atención de los administrativos y su vinculación con el área académica.

Una educación será de calidad, en la medida en que la institución pueda reconocer y ponderar las demandas de sus estudiantes, acordando un compromiso, para satisfacer plenamente sus necesidades educativas y se le ayude a desarrollar al máximo sus posibilidades personales (Senlle \& Gutiérrez, 2004), de tal modo que pueda satisfacer las expectativas generadas en los estudiantes. Cabe resaltar, que la satisfacción se da cuando el estudiante realiza una comparación entre sus expectativas acerca del servicio que va a recibir y el producto educativo dado por la universidad.

Vergara \& Quesada, 2011 concluyen que para poder aumentar la calidad del servicio académico percibido se debe hacer énfasis en las percepciones de los servicios que tienen los estudiantes. También aumentar la calidad del servicio académico tendrá un doble efecto positivo sobre la satisfacción de los estudiantes de la facultad, incrementando el valor percibido por el estudiante y su satisfacción. Luego, por la relación que existe entre el valor percibido por el estudiante y la satisfacción del mismo, se observa que si aumenta el valor percibido por el estudiante, aumentará su satisfacción hacia los servicios que ofrece la institución.

Diversas investigaciones han señalado que uno de los factores más relevantes de la satisfacción estudiantil está relacionado con el proceso de enseñanza-aprendizaje segun (Egea, 2001), por ello muchas instituciones han centrado sus esfuerzos en evaluar sólo está área y se han preocupado por generar las mejoras educativas, sólo desde este punto de vista, sin considerar los otros aspectos que engloba la calidad educativa y que permitirían tener una visión de conjunto.

El análisis del diagnóstico situacional de la UAP, en relación a la segunda variable, satisfacción laboral docente, dio como resultado general, un grado de satisfacción regular en $89.1 \%$ y una satisfacción baja en un 10.9\%; los docentes no muestran un grado de satisfacción alta, y esto se refleja principalmente en la dimensión labor docente-gestión, en un $56.4 \%$ de satisfacción regular y $43.6 \%$ de satisfacción baja. Es decir existe poca participación de los docentes en el sistema de gestión de calidad, no están 
En la investigación realizada por (Rodríguez \& Vélez, 2004), el nivel en el que se ha obtenido el mejor grado de satisfacción (65.3\% BS) ha sido el nivel 3 Satisfacción por el tipo de relación establecida, que supone todo lo referente a las relaciones interpersonales y a sentirse aceptado dentro de un grupo. Este nivel correspondería a las necesidades sociales dentro de la jerarquía de necesidades humanas de Maslow (1954) y tiene como único componente el área 7 Trato respetuoso.

Según Guillén \& otros, 2000 la satisfacción laboral depende del grado de coincidencia, entre lo que una persona quiere y busca en su trabajo y lo que le reporta. A mayor distancia en sentido descendente entre lo deseado y lo encontrado, menor satisfacción. Estas distancias pueden producirse al comparar aspectos intrapersonales, de allí, que la motivación laboral para realizar trabajo en grupo, es un elemento favorecedor sustancialmente del clima organizacional, cuando en las instituciones educativas se observa dificultad para realizar trabajo en grupos se aprecia un distanciamiento entre los individuos que conforman el capital intelectual de la institución.

\section{CONCLUSIONES}

En la medida que se mejora la satisfacción laboral docente, también mejora la calidad educativa, además las opiniones están ubicadas de acuerdo a los descriptivos en Calidad Educativa en mayor porcentaje en Medianamente eficiente y en Satisfacción Laboral Docente en Regular; por tanto guardan también una relación cualitativa directa entre dichas variables de estudio.

La percepción de los estudiantes frente a la calidad educativa de la UAP, fue que el $96.9 \%$ considera medianamente eficiente y solo $3.1 \%$ considera eficiente. Y la percepción de los docentes frente a la satisfacción laboral en la UAP, muestra que el $89.1 \%$ de los docentes se encuentra regularmente satisfecho en su labor docente y solo un $10.1 \%$ el grado de satisfacción es bajo.

La percepción de los estudiantes frente a la calidad educativa de la UAP, en la dimensión de Gestión Universitaria fue la que obtuvo más bajo calificativo ya que el $66.3 \%$ la consideró deficiente y el $33.7 \%$ medianamente eficiente. Mientras que la percepción de los estudiantes frente a la calidad educativa de la UAP, en la dimensión de formación profesional fue que el $99.4 \%$ considera medianamente eficiente y el $0.6 \%$ eficiente.

La percepción de los docentes frente a la satisfacción laboral en la UAP, en la dimensión de Labor docente-gestión, fue que el $56.4 \%$ de los docentes se encuentra regularmente satisfecho y el $43.6 \%$ en un nivel de satisfacción bajo. Mientras que la satisfacción laboral de los docentes en la dimensión de Labor de enseñanza y reconocimiento, fue que el $100 \%$ de los docentes siente un alto grado de satisfacción.

\section{AGRADECIMIENTO:}

A los estudiantes y docentes de la Universidad Alas Peruanas Filial Jaén por su colaboración por responder los instrumentos que brindó información real para esta investigación y aportar el proceso de acreditación en la UAP, Filial Jaén.

\section{BIBLIOGRAFIA}

Atalaya, M. (1995). Satisfacción Académico Administrativa en estudiantes de PostGrado Lima UNMSM. Recuperado de h t t p : / / w w w . u n m s m . e d u . pe / archivos/ocaa/normativas/modelo_calid ad_acreditacion_universitaria.pdf.

Arguelles, D. \& Angles, N. (2004) Estrategias para promover procesos de aprendizaje autónomo. Ed. Contacto. Bogotá, Colombia. 2004

Avila, H. (2010). Metodología de la Investigación. (1ra Edición). Lima. Perú. 
Barrera, C., Gómez, J. \& Hernández, F. (2003). Percepción de la satisfacción de los estudiantes de pregrado acerca de su programa académico, F.N.S.P, Medellín Demografía y Salud: Grupo de Investigación. Recuperado el 20 de s e t i e m b r e, $\quad 2007$ d e http://guajiros.udea.edu.co/demografiay salud/Documentos/percepcion estudiantes pregrado_fnsp_mllin_2003. pdf.

Chiavenato, I. (2001). Proceso de la gestión Administrativa. Tercera edición.

Colombia.

DEA-CONEAU (2011). Modelo de Calidad para la Acreditación de las Carreras Profesionales Universitarias. D i s p o n i b l e e $n$ http://www.coneaces.gob.pe.

Egea, M. (2001). Criterios de calidad en centros universitarios según el alumnado: implicaciones laborales y organizacionales. Revista de Psicología del Trabajo y de las Organizaciones., 17(2), 219.

Guillén, \& otros. (2000). Psicología del trabajo para las Relaciones Laborales. México: McGraw Hill.

Rodríguez, M., \& Vélez, L. (2004). Satisfaccion de los graduandos con la formacon recibida en la Corporación Universitaria Lasallista. REvista Lasallista de Investigación, 55.

Senlle, A., \& Gutiérrez. (2004). Calidad de los Servicios Educativos . Madrid.

SINEACE, M. (2008). www.sineace.gob.pe. Recuperado el 15 de setiembre de 2015, de Reglamento de la Ley $N^{\circ} 28740$, Ley del Sistema Nacional de Evaluación, Acreditación y Certificación de la C a $1 \mathrm{i} \mathrm{d}$ a d E d u c a $\mathrm{t}$ i va : http://repositorio.sineace.gob.pe/reposit orio/handle/123456789/1462.
Sosa, S. (2011). Calidad en los servicios Educativos en la Universidad de San Martín de Porres Filial Norte. Chiclayo: Universidad de San Martín de Porres.

Vergara, J., \& Quesada, V. (2011). Análisis de la calidad en el servicio y satisfacción de los estudiantes de Ciencias Económicas de la Universidad de Cartagena mediante un modelo de ecuaciones estructurales. Cartagena: Universidad de Cartagena.

Viaña, J., Vargas, A., \& Golergant, J. (30 de $\mathrm{N}$ o v e i m b r e de 20007 ). www.coneau.gob.pe. Recuperado el 15 de Setiembre de 2015 , de http://www.upao.edu.pe/oca/pdf/Tomo_ VI_Estandares_para_la_Acreditacion_d e_Farmacia_y_Bioquimica_Tecnologia _Medica_y_Ciencias_Biologicas.pdf.

\section{CORRESPONDENCIA}

Delicia Liliana Bazán Tantaleán

Calle Las Diamelas N 111 - Jaén

lideli109@gmail.com

dbazantantalean@unj.edu.pe 